Fixed Point Theory, 18(2017), No. 1, 293-304

http://www.math.ubbcluj.ro/ nodeacj/sfptcj.html

\title{
OPTIMAL GRONWALL LEMMAS
}

\author{
NICOLAIE LUNGU* AND SORINA ANAMARIA CIPLEA** \\ *Technical University of Cluj-Napoca, Department of Mathematics \\ 28 Memorandumului Street, 400114 Cluj-Napoca, Romania \\ E-mail: nlungu@math.utcluj.ro \\ ** Technical University of Cluj-Napoca, Department of Management and Technology \\ 28 Memorandumului Street, 400114 Cluj-Napoca, Romania \\ E-mail: sorina.ciplea@ccm.utcluj.ro
}

\begin{abstract}
In this paper we give some results in connection with Problem 5 and Problem 9 in $[22$, p. 223 and p. 226]. Here we consider some optimal Gronwall lemmas, consequences of Abstract Gronwall Lemma [20] and some Gronwall lemmas which are not optimal Gronwall lemmas (see [22, Example 6.2, p. 224] or [5, p. 226]).
\end{abstract}

Key Words and Phrases: Gronwall lemmas, optimal Gronwall lemmas, abstract Gronwall lemma, Picard operators, Volterra integral inequalities.

2010 Mathematics Subject Classification: 45G10, 45M10, 45N05, 47N10, 47H10.

\section{REFERENCES}

[1] D. Bainov, P. Simeonov, Integral Inequalities and Applications, Mathematics and its Applications, 57, Kluwer Academic Publishers Group, Dordrecht, 1992.

[2] A. Corduneanu, An integral inequality in two independent variables, An. Ştiinţ. Univ. "Al. I. Cuza" Iaşi, Secț. Ia, Matematică, 29(1983), 69-74.

[3] A. Corduneanu, A nonlinear integral inequalities in two independent variables, An. Ştiinţ. Univ. "Al I. Cuza" Iaşi, Secţ. Ia, Matematică, 30(1984), 5, 15-18.

[4] A. Corduneanu, Integral inequalities in two independent variables, Rev. Roum. Math. Pur. Appl., 32(1987), no. 4, 331-341.

[5] C. Crăciun, N. Lungu, Abstract and concrete Gronwall lemmas, Fixed Point Theory, 10(2009), no. 2, 221-228.

[6] V. Lakshmikantham, S. Leela, Differential and Integral Inequalities: Theory and Applications, Academic Press, New York - London, 1969.

[7] V. Lakshmikantham, S. Leela, A.A. Martynyuk, Stability Analysis of Nonlinear Systems, Marcel Dekker, New York, 1989.

[8] N. Lungu, On some Gronwall-Bihari-type inequalities, Libertas Mathematica, 20(2000), 67-70.

[9] N. Lungu, On some Gronwall-Bihari-Wendorff-type inequalities, Seminar on Fixed Point Theory, 3(2002), 249-254.

[10] N. Lungu, Qualitative Problems in the Theory of Hyperbolic Differential Equations, Digital Data, Cluj-Napoca, 2005.

[11] N. Lungu, On some Volterra integral inequalities, Fixed Point Theory, 8(2007), no. 1, 39-45.

[12] N. Lungu, C. Crăciun, Pseudo-parabolic inequalities, Carpathian J. Math., 27(2011), no. 2, 201-207. 
[13] N. Lungu, I.A. Rus, Hyperbolic Differential Inequalities, Libertas Mathematica, 21 (2001), 3540.

[14] N. Lungu, I.A. Rus, On a functional Volterra-Fredholm integral equation, via Picard operators, J. Math. Ineq., 41(2009), 519-527.

[15] N. Lungu, I.A. Rus, Gronwall inequalities via Picard operators, An. Ştiinţ. Univ. "Al. I. Cuza" Iaşi, Secţ. I, Matematică, LVIII(2012), no. 2, 269-278.

[16] A.A. Martynyuk, V. Lakshmikantham, S. Leela, Stability of Motion: Method of Integral Inequalities, Nauk. Dumka, Kiev, 1989.

[17] D.S. Mitrinović, J.E. Pečrić, A.M. Fink, Inequalities Involving Functions and Their Integrals and Derivatives, Kluwer, 1991.

[18] B.G. Pachpatte, Inequalities for Differential and Integral Equations, Academic Press, New York - London, 1998.

[19] I.A. Rus, Weakly Picard operators and applications, Seminar on Fixed Point Theory, ClujNapoca, 2(2001), 41-58.

[20] I.A. Rus, Picard operators and applications, Sci. Math. Jpn., 58 (2003), no. 1, 191-219.

[21] I.A. Rus, The theory of a metrical fixed point theorem: theoretical and applicative relevance, Fixed Point Theory, 9(2008), 541-559.

[22] I.A. Rus, Gronwall Lemmas: Ten open problems, Sci. Math. Jpn., 70(2009), no. 2, 221-228.

[23] W. Rundell, M. Stecher, Remarks concerning the support solutions of pseudoparabolic equations, Proc. Amer. Math. Soc., 63(1977), no. 1, 77-81.

Received: June 12, 2014; Accepted: December 11, 2014. 